\title{
Gamificação como Tecnologia Educacional Auxiliadora no Ensino de Curativos
}

\author{
Kátia Carola S. Silva1, Mariana Boulitreau S. C. Barros1, Valdir Alfredo O. da \\ Silva2, Luiz Miguel P. Sanches1, Joel Azevedo de M. Netoz, Sean Jasube de $O$. \\ Alves1
}

1 Universidade Federal de Pernambuco-Centro Acadêmico de Vitória (UFPE/CAV)

R. Alto do Reservatório, S/n - Bela Vista, Vitória de Santo Antão - PE, 55608-680

${ }_{2}$ Universidade Federal Rural de Pernambuco (UFRPE)

Rua Dom Manuel de Medeiros, s/n - Dois Irmãos, Recife - PE, 52171-900

3Sociedade Brasileira de Enfermagem em Feridas e Estética

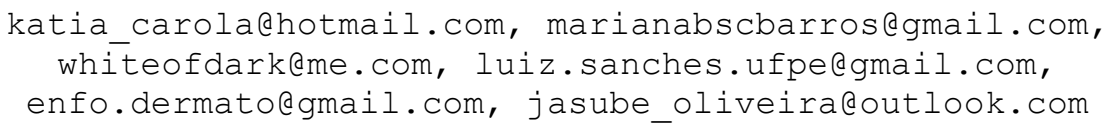

\begin{abstract}
The present year seeks to describe an experience in the construction of a serious game, as well as its potential as a facilitator for the accomplishment of a seminar related to the accomplishment of dressings in students of the nursing course. It is an experience report for the development of a five-step game: 1) Survey of application requirements; 2) Content construction; 3) Creation of the prototype of screens; 4) Game development; 5) Evaluation of the dynamics of the game. The game "Simulator of Attention to Injuries" presents as main scenario an infirmary in hospital scope. It consists of clinical cases of increasing size of difficulty Composed of a simple layout containing the top of the screen of the Nursery, Nursery, and Bed.
\end{abstract}

Resumo. O presente estudo busca descrever a experiência na construção de um jogo sério, bem como sua potencialidade como ferramenta metodológica auxiliadora para o desenvolvimento da semiotécnica relacionada à realização de curativos em discentes do curso de enfermagem. Trata-se de um relato de experiência para o desenvolvimento de um Jogo Sério em cinco etapas: 1) Levantamento dos requisitos da aplicação; 2) Construção do conteúdo; 3) Criação do protótipo de telas; 4) Desenvolvimento do jogo; 5) Avaliação da dinâmica do jogo. O jogo "Injury Care Simulator" apresenta como cenário principal uma enfermaria em âmbito hospitalar. É constituído por casos clínicos de níveis crescente de dificuldade Composto por um layout simples contendo no canto superior da tela o Prontuário, Enfermaria, Loja e o Leito. 


\section{Introdução}

Ao longo tempo, diferentes concepções de natureza filosóficas, cientificas e religiosas emergiram em relação ao corpo humano, onde o corpo ferido e seus métodos de cura foram e continuam sendo alvo eminente de questionamentos (GEOVANINI, 2014). As Feridas são evidenciadas pela ruptura da pele, podendo ser acompanhadas por lesões em músculos, tendões e ossos, classificadas quanto sua etiologia, evolução, complexidade, comprometimento tecidual, espessura e presença de infecção (SMANIOTTO et al, 2012; GEOVANINI, 2014). Perante as estratégias empregadas atualmente para o tratamento das feridas, o método clínico executado mediante a realização do curativo, é o mais utilizado (SMANIOTTO et al, 2010). Curativo ou cobertura se caracteriza como meio terapêtico pelo qual ocorre a realização do tratamento no local da lesão, consistindo na limpeza e aplicação de material, objetivando sua proteção, absorção e drenagem, auxiliando desta forma seu processo de cura (BELLINASO et al, 2014 apud SILVA, 2017).

A realização da assistência para pacientes com feridas é vista atualmente como responsabilidade conjunta, devendo ser efetivada através de um processo dinâmico e complexo através do acompanhamento multidisciplinar, no entanto, diante da periodicidade de contato com o paciente, a equipe de enfermagem passou a desenvolver uma significativa parcela de responsabilidade no tratamento das feridas, onde sua prática assistencial para essas pessoas é um desafio que necessita conhecimentos específicos, habilidades e abordagem holística para fundamentar essa prática. (GEOVANINI, 2014; SILVA ET AL, 2017). Considerando o exposto, a formação acadêmica é crucial para a construção das bases dessas competências, necessitando de uma formação que apoie a articulação da teoria e a prática (BARATIERI et al, 2015).

Com o intuito de romper o paradigma baseado na utilização de metodologias tradicionais, jogos e elementos de gamificação estão sendo inseridos como estratégia facilitadora no ensino e aprendizagem, adquirindo caráter motivador para os estudantes da saúde (GURGEL e FERNANDES, 2015; CASTRO e GONÇALVES, 2018).

Esses jogos, apresentando aspecto auxiliador nesse processo são denominados jogos sérios (JSs) (ROCHA et al, 2016)Baseando a gamificação, na utilização da dinâmica dos jogos aplicados em circunstâncias diferentes do contexto esperado, apresenta intuito de contribuir para a resolução de diferentes problemas. Tendo a capacidade de proporcionar incentivo ao desempenho da aprendizagem ativa, a aprendizagem experiencial e a aprendizagem baseada em problemas (MARCHIORI, 2016; FRAGELLI, 2017).

Partindo desse pressuposto, a Tecnologia da Informação e Comunicação (TIC) presente por meio do jogo eletrônico, apresenta-se como uma forte contribuição para melhoria da qualidade do processo formativo e, desse modo, favorecendo a qualidade da assistência que estes futuros profissionais prestarão. Diante do exposto e da importância em explorar tal tema, o presente estudo tem o objetivo de descrever a experiência na construção de um jogo sério, bem como sua potencialidade como ferramenta metodológica auxiliadora para o desenvolvimento da semiotécnica relacionada à realização de curativos em discentes do curso de enfermagem 


\section{Fundamentação Teórica}

A realização da assistência para pacientes com feridas é vista atualmente como responsabilidade conjunta, devendo ser efetivada através de um processo dinâmico e complexo por meio do acompanhamento multidisciplinar, no entanto, diante da periodicidade de contato com o paciente, a equipe de enfermagem passou a desenvolver uma significativa parcela de responsabilidade no tratamento das feridas, onde sua prática assistencial para essas pessoas é um desafio que necessita conhecimentos específicos, habilidades e abordagem holística para fundamentar essa prática (GEOVANINI, 2014; SILVA ET AL, 2017).

Nesse sentido, a enfermagem apresenta autonomia perante a prática de cuidados a pacientes portadores de feridas, garantida através da resolução COFEN n ${ }^{\circ}$ 567/2018, além de possuir especialidade dentro da enfermagem, reconhecida pela Sociedade Brasileira de Enfermagem Dermatológica (SOBEND) e Associação Brasileira de Estomaterapia (SOBEST) (FERREIRA et al, 2008).

Sendo a formação acadêmica crucial para a construção das bases dessas competências, necessitando de uma formação que apoie a articulação da teoria e a prática (BARATIERI et al, 2015). Com o intuito de romper o paradigma baseado na utilização de metodologias tradicionais, jogos e elementos de gamificação estão sendo inseridos como estratégia facilitadora no ensino e aprendizagem e motivadora para os estudantes da saúde (GURGEL e FERNANDES, 2015; CASTRO e GONÇALVES, 2018).

Baseando a gamificação, na utilização da dinâmica dos jogos aplicados em circunstâncias diferentes do contexto esperado, apresenta intuito de contribuir para a resolução de diferentes problemas. Tendo a capacidade de proporcionar incentivo ao desempenho da aprendizagem ativa, a aprendizagem experiencial e a aprendizagem baseada em problemas (MARCHIORI, 2016; FRAGELLI, 2017). Partindo desse segmento, o entrelaçamento da aprendizagem significativa e a estratégia para o docente o jogo surge como instrumento motivacional (FRAGELLI, 2017).

\section{Caminhos Metodológicos}

Trata-se de um relato de experiência, integrando o processo de desenvolvimento de uma tecnologia móvel no formato de jogo sério, elaborado a partir do interesse em integrar os conteúdos em enfermagem relacionados ao cuidado com curativos em uma ferramenta diferenciada, saindo do método tradicional, rígido e mecânico de transmitir o conhecimento.

O estudo está sendo realizado na Universidade Federal de Pernambuco- Centro Acadêmico de Vitória de junho de 2018 até o momento, por meio da integração de discentes da Universidade Federal de Pernambuco (UFPE) e Universidade Federal Rural de Pernambuco (UFRPE), tendo como coordenação o Núcleo de enfermagem da UFPE. Seu processo de criação envolve cinco etapas, no qual estão constituídas por: 1) Levantamento dos requisitos da aplicação; 2) Construção do conteúdo; 3) criação do protótipo de telas; 4) Desenvolvimento do jogo; 5) avaliação da dinâmica do jogo. Sendo a quarta etapa em processo de desenvolvimento.

Todo desenvolvimento está sendo realizado por uma equipe composta por estudantes do curso de enfermagem, ciências biológicas e licenciatura em informática, 
tendo como coordenadores do projeto os professores da disciplina de informática aplicada à saúde. Além de suporte técnico de um enfermeiro especialista em feridas e curativos.

Na primeira etapa do desenvolvimento do JSs, denominado pelos autores "Injury Care Simulator" foi realizado de maneira sucinta, o levantamento dos requisitos da aplicação do game em sala de aula, atendendo aos aspectos que englobam as necessidades dos discentes e docentes, atrelando simulação, aprendizagem e pensamento crítico. Deste modo, com o intuito de abranger a realidade do discente de enfermagem em sua prática hospitalar foram definidos os temas e as habilidades que integrariam as situações-problema de cada cenário. $\mathrm{Na}$ tabela 1 a seguir, pode-se observar algumas temáticas abordadas.

Tabela 1 - Temática que constitui os cenários do Jogo sério "Injury Care Simulator". Vitória de Santo Antão, Pernambuco, Brasil, 2019.

\begin{tabular}{|l|l|}
\hline Fase & Temática abordada \\
\hline 1 & $\begin{array}{l}\text { Cateter venoso periférico - dispositivos de acesso venoso usado para a } \\
\text { administração de medicamentos, nutrientes ou líquidos na veia. }\end{array}$ \\
\hline 2 & Escoriações - são lesões simples, que ocorrem na camada mais superficial da pele \\
\hline 3 & $\begin{array}{l}\text { Lesão por pressão Estágio 1- Lesão na pele e no tecido subjacente resultante da } \\
\text { pressão prolongada, podendo ser classificada em estágios de acordo com camada } \\
\text { na pele lesada. }\end{array}$ \\
\hline 4 & Doenças cutâneas-Bolhosas - Doenças na qual atinge a pele formando bolhas \\
\hline $5,5.1$ & Mordeduras/ Animais Peçonhentos \\
\hline
\end{tabular}

As temáticas para os respectivos cenários foram escolhidas mediante pesquisa na literatura e elencadas de acordo com níveis de dificuldade.

A segunda etapa, foi marcada pela construção do conteúdo do JSs, constituído por casos clínicos, referente a cada cenário do game. Ex: fase 1: Paciente M.C.G do sexo feminino, 33 anos, foi admitida no hospital apresentando episódio de dor abdominal em uma escala de 5+/10+. Após consulta médica, foi realizada administração de dipirona juntamente no Soro Fisiológico. Os casos clinicos foram elaboras a partir de vivência prática por professores especialistas na área e posteriormente validado pelo professor especialista da Universidade Federal de Pernambuco.

A terceira etapa foi constituída pela formulação do protótipo das telas do JSs, composto pelo seu desenho, sua dinâmica e pelos materiais necessários à ambientação com a trama. Essa etapa foi desenvolvida utilizando imagens do banco de vetores grátis disponíveis na web e estruturada no powerpoint, onde foi possível construir a modelagem inicial, bem como os botões interativos que estarão presentes, no qual poderão sofrer alteração ao longo do projeto. Também foram utilizadas imagens provenientes do acervo de um especialista membro da Sociedade Brasileira de Enfermagem em feridas e estética.

Após o processo de prototipagem e planejamento foi iniciado o desenvolvimento do JSs, iniciando a quarta etapa.

Com a viabilidade de desenvolver uma aplicação móvel compatível com Android, o JSs está sendo construído a partir do Android Studio. Este software está sendo responsável por desenvolver o protótipo por completo, desde sua programação, 
quanto a edição de design. Desenvolvida pelo Google com base no Intelli da JetBrains, esta ferramenta é uma IDE (Integrated Development Environment ou Ambiente de desenvolvimento integrado). Os programas que estão sendo utilizados para desenvolver a estrutura do layout e o design do aplicativo serão o Adobe Experience Design e o Adobe Photoshop. O Adobe Experience Design é uma ferramenta baseada em vetores desenvolvida e publicada pela Adobe Inc. para projetar e criar protótipos de experiência do usuário para aplicativos da Web e móveis. O Adobe Photoshop é um software de edição de imagens e fotos de interface intuitiva e completa, com poderosas ferramentas de edição e manipulação de imagens. Ele oferece a possibilidade de pequenos ajustes nas imagens até edições artísticas de alta complexidade, tendo acesso a inúmeras possibilidades como aplicação de filtros de imagem, uso de camadas, ajustes de cores, entre outros.

A quinta e última etapa do estudo ainda não pôde ser realizada, pois o JSs ainda está no processo de conclusão do desenvolvimento.

\section{Resultados e Discussões}

A motivação pela curiosidade crítica a partir de um tempo altamente "tecnologizado", defende dos "irracionalismos" decorrentes do ou produzidos por certo excesso de "racionalidade", assim como da arrancada falsamente humanista de negação da tecnologia e da ciência. Divinizar ou diabolizar a tecnologia ou a ciência é uma forma altamente negativa e perigosa de pensar errado (Freire, Paulo, 2018).

A partir das etapas que subsidiam o desenvolvimento do game, foi possível construir duas fases do jogo. Apresentando o hospital como cenário principal, o "Injury Care Simulator" introduz o jogador, denominado aprendiz no papel de estagiário, tendo como tutora no ambiente de jogabilidade a enfermeira Fabiane. Ela irá ser responsável por indicar os ícones de interação do jogo. Composto por um layout simples contendo no canto superior da tela o Prontuário, Enfermaria, Loja e o Leito. O jogador tem como objetivo escolher os materiais para realizar o curativo, bem como a ordem de sua utilização de acordo com cada nível de gravidade (figura 1).

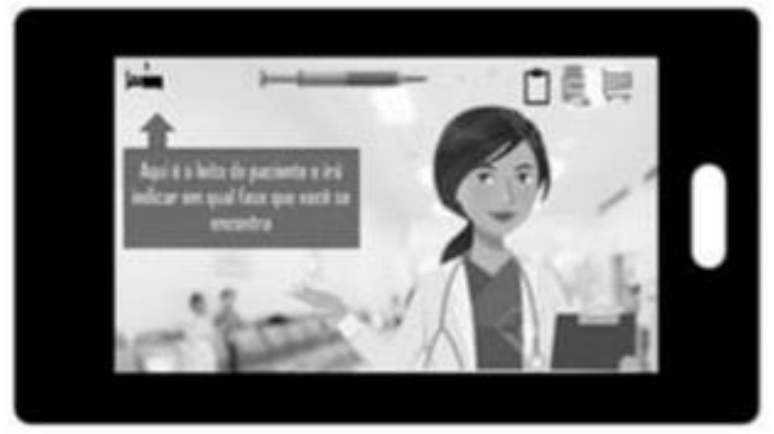

Figura 1. Protótipo de tela, tutora Fabiane indicando a funcionalidade dos ícones do jogo "Injury Care Simulator"

$\mathrm{Na}$ Enfermaria podemos encontrar todos os materiais necessários para realizar a montagem da bandeja e descarte dos materiais utilizados após a realização dos curativos, local fixo presente em todas as fases.

No Prontuário estará localizado o caso clínico, local de registro dos cuidados de enfermagem de extrema importância, onde consta as informações fundamentais para 
realizar a escolha dos materiais e seguir para o atendimento ao paciente que se encontra no leito, ou seja, na respectiva fase do jogo.

A cada acerto, o jogador recebe lamparinas, fazendo alusão ao símbolo da enfermagem, serão recebidas como incentivo ao curativo realizado pelo aprendiz. A quantidade de lamparinas varia de acordo com a escolha e sequência dos materiais corretos para a propedêutica.

$\mathrm{Na}$ loja será possível efetuar a troca de lamparinas por seringas para encher a barra de vida, identificada por uma seringa na região superior central na tela do jogo.

Desta forma, levando em consideração as características elucidadas, o jogo "Injury Care Simulator" converge com os requisitos de JSs, presentes no estudo de Rocha et.al (2016), onde afirma:

\begin{abstract}
Os jogos sérios compreendem múltiplos requisitos: definição dos objetivos de aprendizagem/ treinamento e conteúdo, avaliação do desempenho do aprendiz, fidelidade lógica e fisisica da simulação, não linearidade, com foco no desenvolvimento de conteúdos para engajar o aprendiz, tais como a inclusão de desafios, recompensas níveis e feedback contínuo.
\end{abstract}

Em um estudo de revisão integrativa que identificou os serious game desenvolvidos e/ou validados na área da saúde no Brasil, avaliou a importância destes no estímulo ao aprendizado de forma lúdica, sustentando a relevância das tecnologias educacionais no processo de ensino-aprendizagem para auxiliar na prevenção, diagnóstico, tratamento e reabilitação dos usuários (DEGUIRMENDJIAN,2016).

Levando em consideração à graduação de enfermagem se torna essencial a presença de desafios e fidelidade lógica ao mundo externo, pois permite aos jogadores(aprendizes) realizar uma projeção de sua prática, desta forma evidenciando uma educação problematizadora, onde de acordo com Silva et.al (2014) consiste em na construção do conhecimento por meio de situações problema e ancoradas no processo aprendizagem por descoberta, no qual o aluno torna-se protagonista nesta metodologia.

Um serious game como estratégia na promoção da saúde no combate ao uso de drogas apontou que o uso de jogos digitais tende a ser uma ferramenta de aprendizagem e motivação no contexto educacional, além de promover maior envolvimento dos estudantes (DAMASCENO, 20116).

\title{
5. Conclusão
}

A aprendizagem ativa baseada na utilização de jogos e celulares em sala de aula cada vez mais vem ganhando seguidores, esse processo metodológico de ensino e aprendizagem retira o aluno de um estado de arreflexão e de passividade e por meio de uma Figura 2. Protótipo de tela, prontuário do jogo "Injury Care Simulator" curiosidade criativa do discente emerge a aprendizagem significativa.

O futuro desse estudo consiste em efetuar o desenvolvimento da fase cinco, onde consiste em uma avaliação da dinâmica do jogo por meio de especialistas e alunos

\section{Referências}

GEOVANINI, T. Tratado de feridas e curativos: enfoque multiprofissional. São Paulo. Rideel,2014 
SMANIOTTO, P.H.S; GALLI R; CARVALHO, V.F; FERREIRA, M.C. Tratamento clínico das feridas - curativos. Rev Med (São Paulo). 2010 jul.-dez.;89(3/4):137-41 .

SILVA, A.C.O; FILHO, E.S.D; SOUSA, G.R.S; SILVA, J. F. S; SILVA, A. L; ARAUJO, C. M. S. As principais coberturas utilizadas pelo enfermeiro. Rev. UNINGÁ. Vol.53, n. 2, pp.117-123(Jul-Set 2017).

BARATIERI, T; SANGALETI, C.T; TRINCAUS, M.R. Conhecimento de acadêmicos de enfermagem sobre avaliação e tratamento de feridas. Rev Enferm Atenção Saúde [Online]. jan/jun 2015; 4(1):2-15.

GURGEL PC, FERNANDES MC. Jogos educacionais no ensino da enfermagem em saúde coletiva: relato de experiência. Rev enferm UFPE on line., Recife, 9(9):93203, set., 2015.

CASTRO, T.C; GONÇALVES, L.S. Uso de gamificação para o ensino de informática em enfermagem. Rev. Bras. Enferm. vol.71 no.3 Brasília May/June 2018

MARCHIORI, P. Z. Gamificação, elementos de jogos e estratégia: uma matriz de referência. InCID: R. Ci. Inf. e Doc., Ribeirão Preto, v. 6, n. 2, p. 44-65, set. 2015/fev. 2016.

FERREIRA, A.M; BOGAMIL, D.D.D; TORMENA, P.C. O enfermeiro e o tratamento de feridas: em busca da autonomia do cuidado. Arq Ciênc Saúde 2008 julset;15(3):105-9.

FRAGELLI, T. B. O.Gamificação como um processo de mudança no estilo de ensino aprendizagem no ensino superior: um relato de experiência. Rev. Inter. Educ. Sup. Campinas, SP v.4 n.1 p.221-233 jan./abr. 2017.

ROCHA, R.V; PEDREO, L.Z; BITTENCURT, I.I; ISOTANI,S; ZEM-LOPES, A.M. Metodologia de Desenvolvimento de Jogos Sérios: especificação de ferramentas de apoio open source. Revista Brasileira de Informática na Educação, Volume 24, Número 3, 2016

SILVA, R.S; PAIXÃO, G.P.N; LINS, D.B; JESUS, R.A; PEREIRA. A Estudo de caso como uma estratégia de ensino na graduação: Percepção dos graduandos em enfermagem. Rev Cuid 2014; 5(1): 606-12 Paulo freire - A pedagogia da autonomia: saberes necessários à prática educativa/Paulo Freire $-57^{\mathrm{a}}$ edição Rio de Janeiro: Paz e Terra, 2018

DAMASCENO, E.F; NARDI, P.A; SILVA, A.K.A; FERNADO. L; LOPES, B; FERNANDES, A. M; A Serious Game as a Strategy for Health Promotion in Combating Drug Misuse. J Bras Tele. 2016;4(2):237-245

DEGUIRMENDJIAN, S.C; MIRANDA, F.M; ZEMMASCARENHAS, S.H; HELENA, S. Serious Game developed in health: Integrative Literature Review Juego Serio desarrollado en salud: Revisión Integradora de la Literatura. J. Health Inform. 2016 Julho-Setembro; 8(3):110-16 